\title{
Planificación del recurso hídrico en América Latina y el Caribe
}

\author{
Planning Water Resources in Latin \\ America and the Caribbean
}

Isabel Guzmán-Arias'

Julio Calvo-Alvarado

Fecha de recepción: I I de octubre del 201 I Fecha de aprobación: 30 de julio del 2012

Guzmán-Arias, l; Calvo-Alvarado, J. Planificacion del recurso hídrico en América Latina y el Caribe.

Tecnología en Marcha.Vol. 26, Nº I. Pág 3-18

Máster en Educación Técnica, ingeniera agrícola, profesora e investigadora de la Escuela de Ingeniería Agrícola del Instituto Tecnológico de Costa Rica (ITCR), Estudiante de Doctorado en Ciencias Naturales para el Desarrollo (ITCR-UNED-UNA).

Correo electrónico: iguzman@itcr.ac.cr

2 Ph.D en Manejo de Recursos Naturales y Ciencias del Suelo, Máster en Hidrología Forestal y Silvicultura, ingeniero forestal, profesor e investigador de la Escuela de Ingeniería Forestal y Rector del Instituto Tecnológico de Costa Rica (ITCR).

Correo electrónico: jucalvo@itcr.ac.cr 


\section{Palabras clave}

Planificación, gestión, recurso hídrico, América Latina, agua.

\section{Resumen}

Se destaca el estado actual de los recursos hídricos en los países de América Latina y el Caribe, con énfasis en los aspectos de planificación y gestión. El análisis se basa en los estudios más recientes sobre el tema, a partir de los cuales se aportan elementos como información actualizada y debidamente verificada sobre la realidad legislativa, institucional y organizativa de los países de la región.

La planificación y gestión integrada del agua se ha caracterizado por una gran diversidad de enfoques legales e institucionales, más la presencia de largos debates que anteceden anteproyectos de leyes de aguas y constantes propuestas de reformas a las pocas leyes aprobadas (Dourojeanni \& Jouravlev, 2002). Algunos países, como México y Chile, presentan experiencias más exitosas conducentes a lograr objetivos importantes, sin embargo, aún requieren la continuidad necesaria en el tiempo y la cobertura requerida y por el momento solo constituyen casos aislados.

\section{Key words}

Planning, management, water resources, Latin America, water.

\begin{abstract}
The current status of water resources management and planning in Latin America and the Caribbean countries is described and discussed. The analysis is based on recent studies made on this subject. The available information is updated and duly verified the reality of legislation, institutions and organizations in the countries of the region. Currently planning and integrated management of water resources have been characterized by great diversity of approaches for the legal and institutional aspects. These processes are anticipated by long debates and constant proposals to reform the few approved water laws (Dourojeanni \& Jouravlev, 2002). Some countries like Mexico and Chile have more successful experiences conducive to achieving important goals, however, they still require the necessary continuity in time and coverage and nowadays are only isolated cases.
\end{abstract}

\section{Introducción}

América Latina es una de las regiones del mundo con más recursos hídricos. Sin embargo, su distribución espacial y temporal provoca escasez de agua en extensas zonas del continente, como Argentina, Bolivia, Brasil, Chile, México y Perú (CEPAL, 2007). Esta situación se acentúa aún más cuando se presenta al mismo tiempo la convergencia de otros elementos como la distribución poblacional, la contaminación de fuentes y la creciente demanda de agua por todos los sectores de consumo, entre otros. Consecuentemente, todos los países se encuentran ante el inevitable reto de la planificación para ejercer una eficiente y efectiva administración de este valioso recurso natural. La región enfrenta desafíos constantes en la gestión del recurso hídrico, lo que implica la necesidad de encontrar fórmulas legales e institucionales a nivel de país capaces de prevenir y solucionar los conflictos crecientes por el uso del agua y las crisis creadas por la ocurrencia de fenómenos naturales extremos, tales como sequías o huracanes (Dourojeanni \& Jouravlev, 200 I, García, 1998).

Mientras los conflictos vinculados al agua se incrementan, parece que en algunos países de la región se ha reducido la capacidad relativa que existía para solucionarlos, debido, entre otras razones, a la acelerada modificación del contexto socioeconómico y político y la creciente demanda del recurso. Ante este escenario tan diferente y complejo, es difícil mejorar los sistemas de gestión, más si se agrega la falta de experiencias positivas para diseñar las estrategias que impulsen un modelo de planificación del recurso hídrico. 
En este artículo se aborda el tema de la planificación y gestión de los recursos hídricos de América Latina y el Caribe con los siguientes objetivos:

\section{Objetivo general}

- Analizar el escenario del estado actual y la planificación de los recursos hídricos en América Latina y el Caribe.

\section{Objetivos específicos}

- Resumir a nivel de país y región la oferta (o disponibilidad) y uso actual de los recursos hídricos.

- Describir el contexto regional en relación con la planificación y gestión de los recursos hídricos, con particular énfasis en América Central.

- Profundizar los aspectos positivos de un caso exitoso de planificación y gestión de los recursos hídricos en América Latina y el Caribe.

\section{Metodología}

Para el estudio se consultaron informes y publicaciones recientes relacionadas con el tema de los recursos hídricos de América Latina y el Caribe, específicamente en materia de planificación y gestión, tales como Ballestero et al. 2005, Jouravlev 200 I,
Dourojeanni 2000, CONAGUA 2006 y Valencia, Díaz e Ibarrola 2004. Con base en estas fuentes de información se describe en términos generales el estado actual y el contexto de la planificación de los recursos hídricos, según regiones previamente definidas (figura I). También se discuten los avances logrados tomando como referencia el concepto de "planificación del recurso hídrico" y se selecciona al menos uno de los casos de países con mejores logros.

\section{Resultados y discusión}

El contexto latinoamericano y del Caribe en la planificación de los recursos hídricos.

Disponibilidad de recursos hídricos en la región

En términos generales, América Latina y el Caribe presentan abundantes recursos hídricos en una superficie que constituye apenas el 15\% de la superficie del planeta. Esta área capta casi el 30\% de la precipitación y genera el 33\% de la escorrentía global. Si se considera que la región cuenta con menos del 10\% de la población mundial, se genera una cifra de oferta de agua por habitante de $28000 \mathrm{~m}^{3} / \mathrm{hab} /$ año como media, la cual es muy superior a la media mundial de $8000 \mathrm{~m}^{3} /$ hab/año, la media para África de 6000 m³/hab/año, la media para Europa de

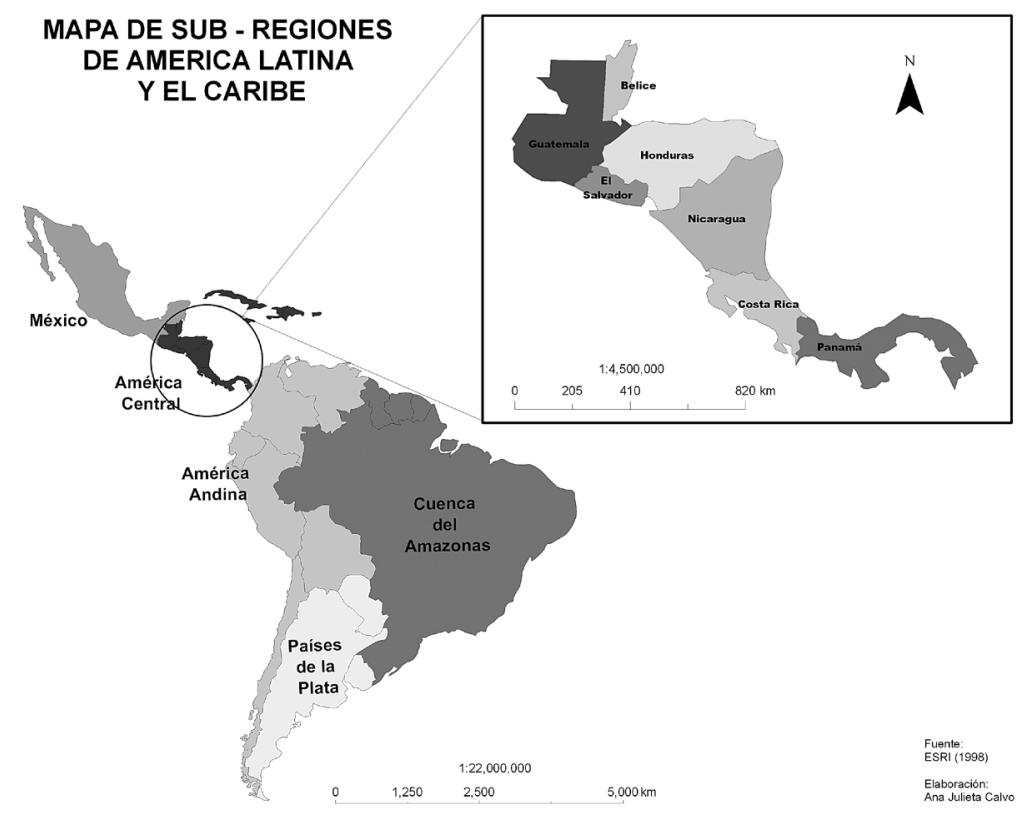

Figura I. Mapa de subregiones de América Latina (Fuente: ESRI, 1998) 
$4000 \mathrm{~m}^{3} / \mathrm{hab} / a n ̃ o$ y la de Asia de $4000 \mathrm{~m}^{3} / \mathrm{hab} / \mathrm{año}$ (FAO, 2000, Ávila, 2002).

La región es húmeda a muy húmeda, con variaciones extremas en cuanto a la disponibilidad geográfica y temporal dentro de un mismo país, entre países y regiones. En México, por ejemplo, cuatro grandes cuencas cubren el $10 \%$ del país y a ellas corresponde el $50 \%$ del caudal medio anual. Por otra parte, tres cuencas en Sudamérica (Orinoco, Amazonas y del Plata) abarcan alrededor de las dos terceras partes del escurrimiento medio anual de toda la región. Casi el 25\% de las tierras en América Latina y el Caribe (unos 5 millones de $\mathrm{km}^{2}$ ) corresponde a regiones áridas y semiáridas como consecuencia de la distribución irregular de las precipitaciones (Aldama \& Gómez, 1996). Estas regiones se encuentran fundamentalmente en el norte y el centro de México, el noreste de Brasil, Argentina y la costa del Pacífico, desde Perú hasta el norte de Chile (el desierto de Atacama en Chile se ha calificado como el lugar más seco del planeta). Se encuentran también zonas más pequeñas en República Dominicana y al norte de Centroamérica (García, 1998).

La distribución de los recursos hídricos dentro de las subregiones muestra situaciones de escasez, que suelen coincidir con las áreas más pobladas de la región. Este es el caso del Valle Central en Chile, la región del Cuyo y el Sur en Argentina, la costa peruana y surecuatoriana, los valles del Cauca y Magdalena en Colombia, el altiplano boliviano, el Gran Chaco, compartido por Bolivia, Argentina y Paraguay, el noreste brasileño, la costa pacífica de América Central y buena parte de México.

En el Cuadro I se presenta la distribución de los recursos hídricos en grandes subregiones, donde se empiezan a evidenciar cifras cercanas al parámetro de escasez generalmente utilizado de $2000 \mathrm{~m}^{3} / \mathrm{hab} /$ año, como es el caso de las Antillas Mayores (FAO, 2000).

\section{Planificación y gestión de los recursos hídricos en América Latina y el Caribe}

De la misma manera que en los países de América Latina y el Caribe existe diversidad en la distribución de los recursos hídricos, existe una enorme variedad de situaciones en su planificación y gestión. Algunos países tienen leyes de reciente aprobación, como México y Brasil, y están en proceso de aplicarlas incluyendo la creación de instituciones como la Agencia Nacional del Agua en Brasil o la masiva creación de Consejos de Cuenca en México; otros llevan años debatiendo leyes de agua y en algunos el tema se ha perdido dentro de la temática ambiental y conflictos institucionales, como es el caso de Costa Rica (Dourojeanni, 2000; Astorga, 2009).

Con este panorama, es difícil dilucidar modelos bien claros de planificación del recurso hídrico en la región, sin embargo, sí existen experiencias de países que han avanzado más en el abordaje de este tema. El Apéndice I resume las características más sobresalientes del entorno legal y administrativo del recurso hídrico en algunos países y para todas las regiones de América Latina.

\section{Planificación y gestión de los recursos hídricos de América Central}

A la fecha, la administración del recurso hídrico en América Central se ha caracterizado por la ausencia de políticas claras, una legislación desactualizada o ausente, traslapes de competencias y funciones entre los entes rectores, supervisores y ejecutores, de carácter público, no público o externo, lo que ha dificultado la administración del recurso hídrico y la toma de decisiones a nivel político. No ha existido una visión de gestión integrada de los recursos hídricos, prevaleciendo las políticas sectoriales (CCAD, CAC \& SISCA, 2006).

Hay que reconocer que dentro de los países centroamericanos han existido diferentes iniciativas con el fin de armonizar las políticas y la legislación en torno a la gestión del agua en la región. Sin embargo, estos esfuerzos no han logrado generar un cambio en la gestión del recurso, que continúa siendo de carácter sectorial, sigue estando supeditada al uso del recurso y sin diferenciar las aguas superficiales de las subterráneas. En ninguno de los países existe una ley específica que regule el manejo de las aguas subterráneas, más bien las leyes y políticas existentes se han establecido para regular usos en forma individual. (Asociación Mundial para el Agua, Centroamérica, 2006)

Actualmente, los países centroamericanos carecen de una política de recursos hídricos y solo Costa Rica (1942), Honduras (1927) y Panamá (1966) han contado con una Ley General de Aguas, leyes que no se crearon con una visión de gestión integrada. A partir del año 2008, Nicaragua cuenta con la Ley General Nacional de Aguas y Honduras, desde 
Cuadro I. Distribución regional de los Recursos Hídricos Internos Renovables (RHIR)

\begin{tabular}{|c|c|c|c|c|}
\hline \multirow[b]{2}{*}{ Subregión } & \multicolumn{2}{|c|}{ Precipitación anual } & \multicolumn{2}{|c|}{ Recursos Hídricos Internos Renovables } \\
\hline & $\mathrm{Mm}$ & $\mathrm{km}^{3}$ & $\mathrm{~km}^{3}$ & $\begin{array}{c}\mathrm{m}^{3} \text { por habitante } \\
\text { (1997) }\end{array}$ \\
\hline México & 772 & 1512 & 409 & 4338 \\
\hline América Central & 2395 & & & 20370 \\
\hline Guatemala & 2200 & & & 8857 \\
\hline Honduras & 1880 & & & 13776 \\
\hline El Salvador & 1180 & 1194 & 6889 & 2755 \\
\hline Nicaragua & $1000-4000$ & & & 34672 \\
\hline Costa Rica & 3300 & & & 27967 \\
\hline Panamá & 3000 & & & 49262 \\
\hline Antillas Mayores & $|45|$ & 288 & 82 & 2804 \\
\hline Antillas Menores & $1|4|$ & 17 & 4 & - \\
\hline Subregión Guayanesa & $|42|$ & 897 & 329 & 191422 \\
\hline Subregión Andina & 1991 & 9394 & 5186 & 49902 \\
\hline Brasil subregión cuenca del Amazonas & 1758 & 15026 & 5418 & 33097 \\
\hline Subregión Sur (Subregión Río de la Plata) & 846 & 3488 & 1313 & 22389 \\
\hline América Latina y el Caribe & 1556 & 31816 & 13429 & 27673 \\
\hline Mundo & & 110000 & 41022 & 6984 \\
\hline Asia (2002) & & - & - & 3920 \\
\hline África (2002) & & - & - & 5720 \\
\hline Europa (2002) & & - & - & 4230 \\
\hline América Latina y Caribe como \% del mundo & & 29 & 33 & \\
\hline
\end{tabular}

Fuente: FAO, 2000, Asociación Mundial para el Agua, Centroamérica, 2006.

2009, dispone de una nueva Ley de Aguas, tal como se muestra en el Apéndice I.

\section{Conceptualización y situación de la planificación de recursos hídricos en América Latina y el Caribe}

La planificación consiste en la definición lógica de una serie de estrategias que permitirán organizar y dirigir la gestión integrada del recurso hídrico. Estas estrategias debidamente documentadas constituyen un plan y son el resultado de un proceso de análisis, toma de decisiones y diseño de esas estrategias con sus respectivas actividades, para alcanzar objetivos predeterminados por y para un grupo relativamente grande de actores que dependen y comparten un mismo recurso y territorio (Dourojeanni, 2000).

Cuando se habla de planificación del recurso hídrico, las decisiones deben tomarse por medio de esquemas participativos establecidos legalmente para que tengan validez y aceptación. Los planes deben considerarse instrumentos de gestión al servicio de los actores. Deben ser conciliatorios en términos económicos, sociales y ambientales, complementarios a los objetivos de cada actor así como del conjunto de actores y no deben suplantar su poder de decisión. Además, es importante que cuenten con la flexibilidad adecuada para que puedan reajustarse cada vez que se dispone de nueva información que hace variar las decisiones (Dourojeanni, 2000).

En los casos en que existen planes de ordenamiento de cuencas, se deben formular teniendo en cuenta marcos regulatorios superiores, por ejemplo, en relación con las políticas económicas vigentes. Los planes, a su vez, deben generar marcos regulatorios específicos que permitan su aplicación. Para que los planes tengan esta prerrogativa de carácter jurídiconormativo, deben elaborarse y aprobarse cumpliendo un conjunto de condiciones procesales. Cuando los marcos regulatorios son claros y estables, existe más libertad para que cada sector usuario de los 
recursos de una cuenca pueda trazar sus planes individuales (Dourojeanni, 2000).

Es importante recordar que, hasta la fecha, en los países de la región casi todas las acciones en materia hídrica se han desarrollado con base en planes de uso sectorial del agua, sobre todo orientados a dar prioridad a proyectos de inversión en obras hidráulicas. Se ha obviado la necesidad de realizar planes integrales y tratar de mejorar la eficiencia de uso múltiple del agua y de los sistemas hidráulicos disponibles. Generalmente, un sector usuario poderoso, como la agricultura bajo riego o la generación de energía hidroeléctrica, ha fijado, en muchos casos, por sí solo el rumbo de aprovechamiento del agua en una cuenca y los otros sectores se han acomodado, en menor o mayor grado, al ritmo del proyecto mayor. En su inmensa mayoría, no se han tomado en cuenta ni los aspectos ecológicos ni los sociales. Revertir esta situación y forma de pensar es un asunto de largo plazo (Dourojeanni, 2000, Guzmán, 2008).

\section{El caso de México, un buen esfuerzo de planificación de recursos hídricos}

México es uno de los países que ha logrado avanzar positivamente, tanto en planificación como en gestión del recurso hídrico. Su proceso de cambio se inició desde 1996, cuando la Comisión Nacional del Agua (CONAGUA) dirigió sus esfuerzos hacia un manejo del recurso más eficaz y participativo, logrando establecer mecanismos de participación como los consejos de cuenca y el consejo consultivo de agua, a fin de lograr el uso y aprovechamiento sustentable del recurso con la colaboración creciente de las autoridades locales en sus diferentes niveles y de los usuarios a nivel regional, además de conducir un proceso de funciones operativas para transferirlas a usuarios y gobiernos locales (CONAGUA, 20I0). El proceso mexicano ha obtenido logros importantes:

I. Integrar, depurar y homologar la información asociada con el agua y sus diferentes usos.

2. Identificar la problemática y sus alternativas de solución, así como definir lineamientos estratégicos para el desarrollo hidráulico.

3. Realizar los programas de acción e integrar los escenarios a largo plazo (2000-2025).
4. Priorizar las acciones cada cuatro años e integrar una cartera de proyectos de corto y mediano plazo.

\section{El proceso de planificación en México}

En México, el proceso de planificación ha tenido la característica de haberse desarrollado de abajo hacia arriba, es decir, se realizó partiendo de la percepción local para integrarla a nivel nacional y sirvió para definir la política hidráulica nacional que se concentra en el Programa Nacional Hidráulico (PNH) 200I-2006 (Valencia et al., 2004), la cual quedó sustentada en cinco premisas:

I. El desarrollo del país debe darse en un marco de sustentabilidad.

2. El agua es un recurso estratégico de seguridad nacional.

3. La unidad básica para la administración del agua es la cuenca hidrológica.

4. El manejo de los recursos debe ser integrado.

5. Las decisiones deben tomarse con la participación de los usuarios.

La integración a nivel nacional de este proceso de planificación se evidencia en la armonización de los objetivos nacionales del sector hidráulico con el marco del Plan Nacional de Desarrollo 200 I-2006 (PND), convirtiéndose estos objetivos en decisivos contribuyentes para la consecución de sus objetivos rectores.

Uno de los logros de mayor impacto dentro del proceso de planificación hidráulica fue la integración, para cada una de las 13 Regiones HidrológicoAdministrativas, de los Programas Hidráulicos Regionales 2002-2006; en ellos se plantean los objetivos y las metas por alcanzar en el ámbito regional, así como las estrategias y acciones en materia hidráulica más importantes por realizar para contribuir a la solución de los problemas que se presentan en cada una de ellas, en congruencia con el $\mathrm{PNH}$.

El proceso de planificación hidráulica se ha caracterizado por la gran participación de la sociedad organizada a través de los Consejos de Cuenca, ampliamente demostrada con 2700 reuniones desarrolladas desde 1998 hasta 2003. Los Consejos de Cuenca validaron la información de los Programas Hidráulicos Regionales 2002-2006 y los consideran el documento rector en materia de planeación hidráulica regional. 


\section{Gestión Integrada de los Recursos Hídricos (GIRH)}

El sector hidráulico en México ha evolucionado en los últimos años hacia un manejo integrado del agua, logro que se deriva de la transformación del marco jurídico y del ente administrador a nivel federal; así como de un proceso ordenado de planificación participativa con los usuarios (Valencia et al., 2004).

I. Los pilares fundamentales que sustentan la Gestión Integrada de los Recursos Hídricos $(\mathrm{GIRH})$ son los siguientes:

2. Ley de Aguas Nacionales, publicada en 1992 y reformada en el mes de abril de 2004.

Establecimiento de la Comisión Nacional del Agua, como órgano superior con carácter técnico, normativo y consultivo de la Federación en materia de $\mathrm{GIRH}$, incluyendo la administración, regulación, control y protección del dominio público del agua.

Al establecer el poder legislativo la Ley de Aguas Nacionales (LAN), tomó fuerza el concepto de GIRH en la agenda pública y el reconocimiento de la cuenca hidrológica como base de la Política Hídrica Nacional, lo que permitiría el fortalecimiento de los mecanismos para mantener o restablecer el equilibrio hidrológico en las cuencas del país y el de los ecosistemas vitales para el agua. Específicamente, la LAN define la GIRH como el proceso que promueve la gestión y desarrollo coordinado del agua, la tierra, los recursos relacionados con éstos y el ambiente, con el fin de maximizar el bienestar social y económico equitativamente sin comprometer la sustentabilidad de los ecosistemas vitales.

En todas las regiones de México se han realizado estudios en los cuales se analizan de manera integrada los recursos de la cuenca, incluyendo su balance hídrico y la demanda de agua, su problemática de conjunto, y se diseñan las estrategias necesarias para atenuar los impactos y propiciar el desarrollo sustentable de la cuenca. Estos estudios han proporcionado elementos suficientes que inducen el desarrollo económico y social de la cuenca mediante un manejo sustentable de sus recursos naturales.

La participación de la sociedad en el proceso ha propiciado los medios para su información y participación en el diseño de estrategias que permitan solucionar la problemática y que fomenten un mejoramiento dentro de la cuenca. Esto conduce a que las instituciones de los tres órdenes de Gobierno trabajen en forma conjunta para lograr el desarrollo social, económico y ambiental de la cuenca.

La CONAGUA ha visualizado la GIRH a nivel de cuenca como un proceso en el cual se deben considerar los siguientes aspectos:

I. Usos del agua integrados

2. Interacción agua superficial y subterránea

3. Disponibilidad de agua en cantidad y calidad

4. Relaciones entre el agua y otros recursos naturales de la cuenca

5. Los recursos naturales y su relación con el desarrollo económico y social.

Otro aspecto importante que surge de la GIRH es la transversalidad de las políticas públicas, la cual se puede definir como la conjunción de esfuerzos de diversos organismos de la Administración Pública Federal, Estatal y Municipal en pro de acciones conjuntas para resolver la problemática ligada a una misma zona, y donde la principal virtud radica en que los esfuerzos para solucionar una problemática dada contribuyen en cierta medida a la solución de otras.

Para fomentar la aplicación de la GIRH,la CONAGUA se propuso revalorar su representatividad en el ámbito de las cuencas hidrológicas, regiones hidrológicas y regiones hidrológico-administrativas, para dar paso a los Organismos de Cuenca, los cuales son unidades técnicas, administrativas y jurídicas especializadas con carácter autónomo. Dichos Organismos de Cuenca deben funcionar armónicamente con los Consejos de Cuenca en la consecución de la GIRH en las cuencas y regiones hidrológicas.

\section{Conclusiones}

Sobre el estado del recurso hídrico en América Latina y el Caribe

Aun cuando América Latina y el Caribe constituyen una región rica en recursos hídricos, representando más del 30\% de la disponibilidad de agua mundial, este recurso está distribuido de manera muy irregular. Una gran parte del mismo se encuentra en la subregión de la Amazonia (Perú, Colombia y Brasil), donde estos países tienen una baja densidad poblacional. Mientras que zonas áridas y semiáridas 
como el centro y norte de México, donde vive una gran parte de la población y que son el motor de la actividad económica nacional, sufren de una escasez constante de agua, tanto por cantidad del recurso como por la calidad.

\section{Sobre la planificación y gestión integral del agua}

Existe una gran variedad de esquemas institucionales y legales para la gestión del agua en los países de América Latina y el Caribe. Mientras algunos se encuentran elaborando la reiterada versión de un anteproyecto de una nueva ley de aguas, otros están tratando de modificar la que aprobaron y otros están en proceso de reglamentarla y aplicarla.

La situación práctica de todos los países demuestra una estructura administrativa del sector hídrico compleja y limitante para el logro de una planificación y gestión integrada. En todos los casos, la capacidad real del Estado para regular el uso del recurso y hacer cumplir las normas es muy débil, lo que se agrava por la generalizada desarticulación y sectorización institucional. En muchos casos, los sistemas administrativos están muy fragmentados, con importantes vacíos de gestión y vulnerables a la politización en las actividades técnicas.

En lo positivo, existen experiencias y esfuerzos importantes como los casos de México, Chile y Brasil. El resto de los países analizados ha conocido numerosos intentos de reforma, sin lograr todavía adecuar el marco normativo del sector hídrico, inexistente u obsoleto en la mayoría de ellos (Ballestero et al., 2005), con la naturaleza de los problemas que presenta el aprovechamiento del agua en la actualidad, así como con las concepciones y prácticas de la sociedad.

Son muchas las dificultades que enfrenta la búsqueda de amplios consensos sociales acerca de las reformas del régimen de aguas. A la hora de asumir este tema, se presenta un reduccionismo de criterios, que no necesariamente responden a la naturaleza del objeto en cuestión; cuando algunos intereses relevantes se excluyen del proceso de reforma o cuando un grupo reducido tiene una influencia desproporcionada, o cuando el objetivo que se persigue tiene más que ver con los problemas coyunturales que afectan a los sectores usuarios del recurso que con la búsqueda de una gestión integrada del agua.
El elemento común en el tema de la administración del recurso hídrico en América Latina y el Caribe es una creciente conciencia de la necesidad de promover su planificación y gestión integrada.

En el caso de México, aunque se han alcanzado logros en las diversas fases del manejo del agua, hoy en día el sector hídrico se encuentra ante un nuevo reto: la Gestión Integrada de los Recursos Hídricos. Claro está, la aplicación práctica de este concepto no parte de cero en este caso; existen estructuras, mecanismos y acuerdos, que se han fortalecido a través del proceso de planificación hidráulica iniciado en 1996. Un notable esfuerzo es la consolidación de la participación social a través de los Consejos de Cuenca, misma que se demuestra en la aprobación y validación de los Programas Hidráulicos Regionales 2002-2006 como documento rector de la política hidráulica en las Regiones HidrológicoAdministrativas. Las disposiciones enunciadas en las reformas a la Ley de Aguas Nacionales indican que la política hídrica nacional se sustenta en una gestión integrada de los recursos hídricos por cuenca hidrológica en forma descentralizada e integrada, en donde la acción directa y las decisiones por parte de los actores locales se deben privilegiar.

Sobre el estado, la planificación y la gestión en América Central

En América Central, pese a los esfuerzos realizados por los gobiernos y la sociedad civil para movilizarse hacia la implementación del conjunto de principios y conceptos que forman parte de la nueva gestión hídrica, no se perciben los avances esperados debido a las características que presenta el entorno económico, social e institucional en este terreno prácticamente en todos los países. En síntesis, la condición de los recursos hídricos en América Central se caracteriza por grandes carencias en cuanto a instrumentos políticos, económicos e institucionales.

\section{Recomendaciones}

Ante la importancia que revisten los recursos hídricos para el desarrollo sostenible de las naciones, un modelo de planificación a nivel de país debe tener como mínimo los siguientes elementos (Van Hofwegen \& Jaspers, 2000):

- Concebir el recurso hídrico como un sistema geográficamente definido, del cual forman parte 
las aguas superficiales y subterráneas, junto con todas las características y procesos físicos, químicos y biológicos asociados.

- El espacio geográfico bajo la influencia de una fuente principal del recurso hídrico debe estar delimitado con base en la coherencia funcional entre los aspectos hidrológicos, morfológicos y ecológicos.

- Debe considerarse la importancia que tienen las funciones de uso de los recursos hídricos para su desarrollo y manejo. Si tales funciones se toman en cuenta correctamente durante el proceso de planificación y gestión del sistema hídrico, se puede hablar de un enfoque que busca equiparar los deseos de la sociedad con respecto al uso del agua con el funcionamiento de los sistemas hídricos a través de intervenciones técnicas y legislativas.

- El marco legal, necesariamente debe ser claro, actualizado y proyectarse a las necesidades futuras.

- Debe haber un ente rector y fiscalizador del recurso, capaz de dirigir la labor de las instituciones involucradas.

De cara a la realidad latinoamericana en general y centroamericana en específico expuesta, se recomienda el diseño de una política hídrica comprensiva de los aspectos económicos, sociales, antropológicos, jurídicos y técnicos, que determine la organización del uso del agua con fines de desarrollo económico y social en términos de eficiencia y equidad.

Esta política hídrica debe contar con una base común para los países de manera que exista coherencia a nivel de la región, sobre todo cuando existen cuencas transfronterizas. Esa base estaría dada por los conceptos y principios contenidos en diversos documentos internacionales que están orientados a la gestión integral del recurso, como los Principios de Dublín, entre otros.

A su vez, esta política debería diseñarse considerando los intereses del recurso mismo, es decir, la posibilidad de conservar y mejorar su cantidad y calidad. Además, deberá estar basada en la más amplia participación de todos los sectores de la población, con el fin de conocer, entre otros, los intereses de los segmentos pobres urbanos y rurales en el ámbito local, además de asegurar su acceso al agua, como lo señala CEPAL (2005).

En ese orden de cosas, el siguiente paso sería diseñar e implementar un sistema de administración de los derechos de agua basado en la planificación del recurso hídrico, que parta del conocimiento de la situación real desde el ámbito local.

Es imperativo conocer y generar información sobre oferta y demanda del recurso hídrico por sectores al menos a nivel de cuenca nacional, empleando balances hídricos mensuales y escenarios de demanda alta-media y baja con un horizonte de al menos 25 años. Sin esta información, es prácticamente imposible planificar y gestionar los recursos hídricos. Igualmente necesario es proveer en estos estudios la estimación de las demandas de agua ambiental tales como caudales ambientales y flujos de agua a humedales, entre otros.

Finalmente, el enfoque de manejo del agua es la gestión de las cuencas hidrográficas, lo cual obliga a desarrollar una institucionalidad adecuada, que en los países del istmo inicia su discusión, en la que es recomendable introducir una instancia de resolución de conflictos. Este espacio se hace necesario no solo porque su pertinencia se deriva del enfoque mismo sino porque los conflictos entre usuarios del agua han empezado a ser más frecuentes y álgidos.

\section{Agradecimientos}

A la Vicerrectoría de Investigación y Extensión del Instituto Tecnológico de Costa Rica, por su apoyo al proyecto Valoración y Planificación del Recurso Hídrico en la cuenca alta y canal principal del río Tempisque. Este artículo es parte de los proyectos: "Long-Term Research in Environmental Biology: Streams of the Área de Conservación Guanacaste", conducido por el Stroud Water Research Center con el apoyo de la USA National Science Foundation (DEB 05-16516) y del proyecto TROPI-DRY: "Dimensiones Humanas, Ecológicas y Biofísicas de los Bosques Secos Tropicales"; proyecto desarrollado por una red de investigación respaldada por el Inter-American Institute for Global Change Research (IAI) CRN II \# 02I, y financiada por la US National Science Foundation (Grant GEO-0452325). 
Apéndice I. Estructura administrativa para una gestión integrada del recurso hídrico en algunos países de América Latina y el Caribe

\begin{tabular}{|c|c|c|}
\hline País & $\begin{array}{l}\text { Legislación en materia del agua y } \\
\text { organización territorial }\end{array}$ & Marco Institucional y administrativo del recurso hídrico \\
\hline México & $\begin{array}{l}\text { Legislación: } \\
12 \text { Ley de Aguas Nacionales (1992) y } \\
\text { reformada (2004) } \\
\text { Organización territorial: Estados }\end{array}$ & $\begin{array}{l}\text { 'La Comisión Nacional del Agua (CONAGUA) es la encargada de for- } \\
\text { mular el programa nacional hidráulico y administrar el agua para todos los } \\
\text { usos. Cuenta con gerencias regionales y un Consejo Técnico. } \\
\text { Entidades relacionadas con el recurso hídrico: } \\
\text { 'Comisión Nacional del Agua (CONAGUA), órgano desconcentrado de } \\
\text { la Secretaría de Medio Ambiente y Recursos Naturales. } \\
\text { Comisión Federal de Electricidad } \\
\text { Comisiones Estatales de Agua }\end{array}$ \\
\hline Guatemala & $\begin{array}{l}\text { Legislación: } \\
\text { 1,10 No existe una ley general. El } \\
\text { ordenamiento jurídico comprende } \\
\text { normas en muchas leyes que regulan } \\
\text { aspectos parciales del aprovecha- } \\
\text { miento y conservación del agua. } \\
\text { Organización territorial: } \\
\text { Departamentos }\end{array}$ & $\begin{array}{l}\text { I,10 La administración del agua está conformada por muchas instituciones } \\
\text { centralizadas, descentralizadas, autónomas, territoriales y especiales, pero } \\
\text { ninguna tiene control completo e integral de su gestión y aprovecha- } \\
\text { miento. } \\
\text { El Ministerio de Medio Ambiente y Recursos Naturales es el encargado de } \\
\text { la administración del uso del agua. } \\
\text { Entidades relacionadas con el recurso hídrico: } \\
\text { 1,10 Ministerio de Medio Ambiente y Recursos Naturales (MARN), creado } \\
\text { en } 2000 \text {. }\end{array}$ \\
\hline Honduras & $\begin{array}{l}\text { Legislación: } \\
\text { "'Ley de Aguas (2009) } \\
\text { Organización territorial: } \\
\text { Departamentos }\end{array}$ & $\begin{array}{l}\text { ' El Consejo Nacional del Desarrollo Sostenible (CONADES) se encarga } \\
\text { de asesorar al gobierno en políticas y estrategias de desarrollo, coordinar } \\
\text { las acciones para el medio ambiente, facilitar el diálogo y crear alianzas } \\
\text { entre los diversos sectores. } \\
\text { La Secretaría de Recursos Naturales y Ambiente (SERNA) formula, } \\
\text { coordina, ejecuta y evalúa las políticas relacionadas con la protección y } \\
\text { aprovechamiento de los recursos naturales. Además brinda servicios de } \\
\text { investigación y control de contaminación en todas sus formas. } \\
\text { Entidades relacionadas con el recurso hídrico: } \\
\text { ' Consejo Nacional del Desarrollo Sostenible (CONADES) } \\
\text { ' Secretaría de Recursos Naturales y Ambiente (SERNA) } \\
\text { ' Subsecretaría de Recursos Naturales y Energía. Dentro de ella se } \\
\text { encuentra la Dirección General de Recursos Hídricos. } \\
\text { ' Subsecretaría de Ambiente }\end{array}$ \\
\hline El Salvador & $\begin{array}{l}\text { Legislación: } \\
\text { 'La gran diversidad de leyes inci- } \\
\text { de en la confusión de campos de } \\
\text { acción y en la indefinición de res- } \\
\text { ponsabilidades. } \\
\text { ' Decreto Legislativo No } 233 \text { (1998) } \\
\text { Creación del Sistema Nacional } \\
\text { de Gestión del Medio Ambiente } \\
\text { (SINAMA) } \\
\text { 'Ley del Medio Ambiente (1998) } \\
\text { Organización territorial: } \\
\text { Departamentos }\end{array}$ & $\begin{array}{l}\text { I Existe dispersión y fraccionamiento institucional en la administración } \\
\text { del recurso hídrico, lo que implica ausencia de coordinación, conflictos } \\
\text { de competencia, dualidad de funciones, sin que ninguna institución asuma } \\
\text { con una visión multisectorial las funciones de planificación, fomento y } \\
\text { conservación del agua. } \\
\text { El Ministerio de Medio Ambiente y Recursos Naturales es responsable de } \\
\text { supervisar la disponibilidad y la calidad del agua y de promover el manejo } \\
\text { integrado de cuencas. } \\
\text { Entidades relacionadas con el recurso hídrico: } \\
\text { ' Ministerio de Medio Ambiente y Recursos Naturales (MARN) } \\
\text { I Sistema Nacional de Gestión del Medio Ambiente (SINAMA) }\end{array}$ \\
\hline
\end{tabular}




\begin{tabular}{|c|c|c|}
\hline País & $\begin{array}{l}\text { Legislación en materia del agua y } \\
\text { organización territorial }\end{array}$ & Marco Institucional y administrativo del recurso hídrico \\
\hline Nicaragua & $\begin{array}{l}\text { Legislación: } \\
\text { ' Ley No } 217 \text { Ley General del Medio } \\
\text { Ambiente y los Recursos Naturales } \\
\text { (1996) } \\
\text { ' Ley No } 290 \text { Ley de Organización, } \\
\text { Competencia y Procedimientos del } \\
\text { Poder Ejecutivo (1998) } \\
{ }^{13} \text { Ley N } 620 \text { Ley General Nacional } \\
\text { de Aguas ( } 2008 \text { ) } \\
\text { Organización territorial: } \\
\text { Departamentos }\end{array}$ & $\begin{array}{l}\text { ' En un esfuerzo por mejorar la gestión del agua, fragmentada y carente } \\
\text { de coordinación, el Ministerio del Ambiente y Recursos Naturales } \\
\text { (MARENA) se ha encargado de formular, proponer y dirigir las políticas } \\
\text { nacionales del medio ambiente y el uso sostenible de los recursos } \\
\text { naturales. } \\
\text { La Comisión Nacional de Recursos Hídricos (CNRH) funciona como } \\
\text { instancia de consulta y coordinación intersectorial para la planificación y } \\
\text { administración integral de los recursos hídricos. } \\
{ }^{13} \text { Autoridad Nacional del Agua (ANA), ente rector pendiente de creación. } \\
\text { Entidades relacionadas con el recurso hídrico: } \\
\text { ' Ministerio del Ambiente y Recursos Naturales (MARENA) } \\
\text { Ministerio de Fomento, Industria y Comercio (MIFIC) } \\
\text { Comisión Nacional de Recursos Hídricos (CNRH) } \\
\text { Comisión Nacional del Ambiente }\end{array}$ \\
\hline Costa Rica & $\begin{array}{l}\text { Legislación: } \\
\text { 'Ley de Aguas (1942). La legislación } \\
\text { hídrica es amplia, desarticulada y } \\
\text { obsoleta. } \\
\text { Organización territorial: Provincias }\end{array}$ & $\begin{array}{l}\text { I El Ministerio de Ambiente, Energía y Telecomunicaciones (MINAET) } \\
\text { es el organismo rector de la gestión de los recursos naturales. La } \\
\text { administración y asignación del agua le compete al Departamento de } \\
\text { Aguas, adscrito al Instituto Meteorológico Nacional (IMN) del MINAET. } \\
\text { Existe poca coordinación interinstitucional y cada una actúa bajo una } \\
\text { visión sectorial. } \\
\text { Entidades relacionadas con el recurso hídrico: } \\
\text { ' Instituto Costarricense de Acueductos y Alcantarillados (A y A) } \\
\text { Instituto Costarricense de Electricidad (ICE) } \\
\text { Servicio Nacional deAguas Subterráneas, Riego y Avenamiento (SENARA) } \\
\text { Asoc. Adm. de Sistemas de Acueductos y Alcantarillados Comunales } \\
\text { (ASADAS) } \\
\text { Municipalidades }\end{array}$ \\
\hline Panamá & $\begin{array}{l}\text { Legislación: } \\
\text { 'Carece de un cuerpo legal integral } \\
\text { y ágil, y de amplias facultades de } \\
\text { gestión en instituciones con compe- } \\
\text { tencia sobre el recurso. } \\
\text { 'Ley No 35, por la cual se reglamen- } \\
\text { ta el uso de las aguas (1966) } \\
{ }^{14} \text { Ley } N^{0} 41 \text { Ley General de } \\
\text { Ambiente (1998) } \\
\text { Organización territorial: Provincias }\end{array}$ & $\begin{array}{l}\text { ' La Autoridad Nacional del Ambiente (ANAM) es la entidad autónoma } \\
\text { rectora en materia de recursos naturales y del ambiente. Cuenta con } \\
\text { amplias atribuciones en materia de agua, correspondiéndole la función de } \\
\text { coordinación, fiscalización, planificación, formulación de políticas, asigna- } \\
\text { ción y control de la contaminación. } \\
\text { Entidades relacionadas con el recurso hídrico: } \\
\text { 'Autoridad Nacional del Ambiente (ANAM) } \\
\text { Consejo Nacional del Ambiente, encargado de la política nacional del } \\
\text { ambiente y del uso sostenible de los recursos naturales. } \\
\text { Comisión Consultiva Nacional del Ambiente, órgano de consulta de la } \\
\text { ANAM. }\end{array}$ \\
\hline Barbados & $\begin{array}{l}\text { Legislación: } \\
\text { No se registra } \\
\text { Organización territorial: Parroquias }\end{array}$ & $\begin{array}{l}\text { ' La Autoridad de Aguas de Barbados (BWA) se encarga de la gestión, } \\
\text { asignación y vigilancia de los recursos hídricos y la prestación de servicios } \\
\text { de abastecimiento de agua potable y saneamiento. } \\
\text { Entidades relacionadas con el recurso hídrico: } \\
\text { ' Autoridad de Aguas de Barbados (BWA), corporación adscrita al } \\
\text { Ministerio de Obras Públicas, Transporte y Vivienda. }\end{array}$ \\
\hline
\end{tabular}




\begin{tabular}{|c|c|c|}
\hline País & $\begin{array}{l}\text { Legislación en materia del agua y } \\
\text { organización territorial }\end{array}$ & Marco Institucional y administrativo del recurso hídrico \\
\hline Cuba & $\begin{array}{l}\text { Legislación: } \\
\text { ' Ley de las Aguas Terrestres (1993) } \\
\text { Organización territorial: Provincias }\end{array}$ & $\begin{array}{l}\text { ' El Instituto Nacional de Recursos Hidráulicos (INRH) es el responsable } \\
\text { de dirigir, ejecutar y controlar la aplicación de la política estatal en lo rela- } \\
\text { tivo a los recursos hídricos. } \\
\text { Las Direcciones Provinciales de Recursos Hidráulicos, conjuntamente con } \\
\text { los Complejos Hidráulicos que se le subordinan, constituyen la estructura } \\
\text { territorial de base del INRH. } \\
\text { El Ministerio de Ciencia, Tecnología y Medio Ambiente es el encargado de } \\
\text { establecer acciones que conduzcan al desarrollo sostenible. } \\
\text { Entidades relacionadas con el recurso hídrico: } \\
\text { ' Ministerio de Ciencia, Tecnología y Medio Ambiente } \\
\text { Instituto Nacional de Recursos Hidráulicos (INRH) } \\
7 \text { Consejo Nacional de Cuencas Hidrográficas (CNCH), Consejos de } \\
\text { Cuencas Territoriales (CCT) y Específicos (CCE) }\end{array}$ \\
\hline Jamaica & $\begin{array}{l}\text { Legislación: } \\
\text { 'Water Resources Act (1996) } \\
\text { Organización territorial: Parroquias }\end{array}$ & $\begin{array}{l}\text { ' La Autoridad de Recursos Hídricos, órgano adscrito al Ministerio de } \\
\text { Agua y Vivienda, se encarga de la gestión, planificación, protección, evalua- } \\
\text { ción, investigación y asignación del agua superficial y subterránea. } \\
\text { La Autoridad de Conservación de Recursos Naturales se encarga de la } \\
\text { gestión ambiental, control de la contaminación y la protección de cuencas. } \\
\text { Entidades relacionadas con el recurso hídrico: } \\
\text { ' Autoridad de Recursos Hídricos } \\
\text { ' Autoridad de Conservación de Recursos Naturales }\end{array}$ \\
\hline $\begin{array}{l}\text { República } \\
\text { Dominicana }\end{array}$ & $\begin{array}{l}\text { Legislación: } \\
\text { ' Ley N } 6 \text { de creación del Instituto } \\
\text { Nacional de Recursos Hidráulicos } \\
\text { (INDRHI) (1965). } \\
\text { Ley N }{ }^{\circ} 5852 \text { Ley sobre Dominio de } \\
\text { Aguas Terrestres y Distribución de } \\
\text { Aguas Públicas (1962) } \\
\text { Ley Nº } 64 \text { Ley General sobre Medio } \\
\text { Ambiente y Recursos Naturales } \\
\text { (2000) } \\
\text { Organización territorial: Provincias }\end{array}$ & $\begin{array}{l}\text { ' Existe multiplicidad de organismos con funciones superpuestas en la } \\
\text { institucionalidad del sector de los recursos hídricos. } \\
\text { El Instituto Nacional de Recursos Hidráulicos (INDRHI) es la máxi- } \\
\text { ma autoridad nacional sobre las aguas superficiales y subterráneas. } \\
\text { Desempeña funciones tanto de gestión como de aprovechamiento del } \\
\text { recurso. } \\
\text { Entidades relacionadas con el recurso hídrico: } \\
\text { ' Instituto Nacional de Recursos Hidráulicos (INDRHI) } \\
\text { Juntas Regantes }\end{array}$ \\
\hline Argentina & $\begin{array}{l}\text { Legislación: } \\
\text { 'No existe una legislación nacional } \\
\text { de agua, solo leyes provinciales, } \\
\text { algunas se están reformando. } \\
\text { Organización territorial: Provincias }\end{array}$ & $\begin{array}{l}\text { 'La gestión de los recursos hídricos se caracteriza por un enfoque estric- } \\
\text { tamente sectorial y una fragmentación institucional. No hay coordinación } \\
\text { interinstitucional. } \\
\text { Entidades relacionadas con el recurso hídrico: } \\
\text { ' Subsecretaría de Recursos Hídricos (SSRH), dependiente de la Secretaría } \\
\text { de Obras Públicas del Ministerio de Planificación Federal, Inversión Pública } \\
\text { y Servicios. } \\
{ }^{2} \text { Consejo Hídrico Federal (COHIFE) }\end{array}$ \\
\hline
\end{tabular}




\begin{tabular}{|c|c|c|}
\hline País & $\begin{array}{l}\text { Legislación en materia del agua y } \\
\text { organización territorial }\end{array}$ & Marco Institucional y administrativo del recurso hídrico \\
\hline Bolivia & $\begin{array}{l}\text { Legislación: } \\
\text { ' Ley de Aguas del } 28 \text { de noviembre } \\
\text { de 1906, en su mayor parte fuera de } \\
\text { contexto actual. } \\
\text { Ley de Protección y Conservación } \\
\text { del Medio Ambiente y los Recursos } \\
\text { Naturales del } 27 \text { de abril de } 1992, \\
\text { que promueve la planificación del } \\
\text { agua. } \\
\text { Proyecto de Ley del Agua, } \\
\text { actualmente en debate (200I). } \\
\text { Organización territorial: } \\
\text { Departamentos }\end{array}$ & $\begin{array}{l}\text { 'Todos los sectores involucrados en el uso del agua realizan sus activida- } \\
\text { des independientemente de acuerdo con las leyes sectoriales, sin estable- } \\
\text { cer un criterio común de utilización y prioridades. } \\
\text { Entidades relacionadas con el recurso hídrico: } \\
\text { lEn el Proyecto de Ley de Agua se propone que el Ministerio de } \\
\text { Desarrollo Sostenible y Planificación sea la Autoridad Nacional del } \\
\text { Recurso Agua. } \\
{ }^{3} \text { Viceministerio de Medio Ambiente y Recursos Naturales } \\
{ }^{3} \text { Dirección General de Cuencas Hidrográficas y Suelos }\end{array}$ \\
\hline Brasil & $\begin{array}{l}\text { Legislación: } \\
\text { ' Ley No } 9433 \text { (1997) Ley de Aguas } \\
\text { y Política Nacional de Recursos } \\
\text { Hídricos. } \\
\text { ' Ley No } 9984 \text { (2000), sobre la } \\
\text { creación de la Agencia Nacional de } \\
\text { Aguas. } \\
\text { Organización territorial: Estados }\end{array}$ & $\begin{array}{l}\text { ' La Unión (nivel federal) es titular de lagos, ríos y cualquier corriente de } \\
\text { agua en terrenos de su dominio o que bañen más de un Estado, sirvan de } \\
\text { límites con otros países o se extiendan a territorio extranjero o proven- } \\
\text { gan de él. También es titular del potencial de la energía hidráulica. } \\
\text { Los Estados son titulares de las aguas superficiales o subterráneas, fluyen- } \\
\text { tes, emergentes y en depósito salvo, en este caso, las derivadas de obras } \\
\text { de la Unión. } \\
\text { Entidades relacionadas con el recurso hídrico: } \\
\text { ' Sistema Nacional de Recursos Hídricos, que comprende: I. Consejo } \\
\text { Nacional de Recursos Hídricos, encargado de la gestión del recurso } \\
\text { hídrico, integrado por representantes de los ministerios relacionados al } \\
\text { agua, de los Consejos Estaduales de Recursos Hídricos, de los usuarios del } \\
\text { agua y de las organizaciones civiles relacionadas con los recursos hídricos } \\
\text { y } 2 \text {. Agencia Nacional de Aguas, con autonomía administrativa y financiera, } \\
\text { adscrita al Ministerio del Medio Ambiente, encargada de la implementa- } \\
\text { ción de la Política Nacional de Recursos Hídricos. } \\
{ }^{4} \text { Comité de Cuenca y Agencias de Cuenca (20 I0) }\end{array}$ \\
\hline Chile & $\begin{array}{l}\text { Legislación: } \\
\text { ' Código de Aguas (198I) } \\
\text { ' Ley No } 19300 \text { (1994) Ley de Bases } \\
\text { del Medio Ambiente } \\
\text { Organización territorial: Regiones }\end{array}$ & $\begin{array}{l}\text { 'Dispersión institucional con contradicciones y superposición de com- } \\
\text { petencias. La responsabilidad en la gestión del agua e implementación } \\
\text { del "Código de Aguas" corresponden a la Dirección General de Aguas } \\
\text { (DGA), ente no sectorial, independiente de los sectores usuarios, No } \\
\text { ejecuta el aprovechamiento del recurso hídrico, lo que le permite } \\
\text { desempeñar su rol regulador y normativo con gran imparcialidad. } 5 \text { Sin } \\
\text { embargo, sus facultades son muy limitadas ya que no puede involucrarse } \\
\text { en la distribución de las aguas, en la transacción de derechos de aguas ni } \\
\text { en la resolución de conflictos. Carece aún de una visión integrada de los } \\
\text { recursos hídricos. } \\
\text { Entidades relacionadas con el recurso hídrico: } \\
\text { ' Dirección General de Aguas (DAP) un servicio dependiente del } \\
\text { Ministerio de Obras Públicas (MOP). } \\
\text { 'Dirección de Obras Hidráulicas } \\
{ }^{5} \text { Dirección de Riego } \\
\text { Dirección de Planeamiento. } \\
\text { Depto. de Defensas Fluviales } \\
\text { Algunos organismos dependientes del Ministerio de Agricultura. } \\
\text { Asociaciones de usuarios. }\end{array}$ \\
\hline
\end{tabular}




\begin{tabular}{|c|c|c|}
\hline País & $\begin{array}{l}\text { Legislación en materia del agua y } \\
\text { organización territorial }\end{array}$ & Marco Institucional y administrativo del recurso hídrico \\
\hline Colombia & $\begin{array}{l}\text { Legislación: } \\
\text { ' Ley No } 99 \text { (1993) de creación del } \\
\text { Ministerio del Medio Ambiente y } \\
\text { organización del Sistema Nacional } \\
\text { Ambiental. } \\
\text { Organización territorial: } \\
\text { Departamentos }\end{array}$ & $\begin{array}{l}\text { I Organización por objetivos ambientales y de desarrollo económico, } \\
\text { caracterizada por una fuerte descentralización del poder y un fortale- } \\
\text { cimiento de los esquemas de índole regional. El Ministerio del Medio } \\
\text { Ambiente, Vivienda y Desarrollo Territorial es el organismo rector de la } \\
\text { gestión del medio ambiente y de los recursos naturales renovables. El } \\
\text { Sistema Nacional Ambiental es el encargado del manejo ambiental del país. } \\
\text { Entidades relacionadas con el recurso hídrico: } \\
{ }^{6} \text { Ministerio de Ambiente, Vivienda y Desarrollo Territorial (MAVDT). } \\
\text { Sistema Nacional Ambiental }\end{array}$ \\
\hline Ecuador & $\begin{array}{l}\text { Legislación: } \\
\text { ' Decreto Ejecutivo No } 2224 \text { (1994). } \\
\text { Creación del Consejo Nacional de } \\
\text { Recursos Hídricos (CNRH). } \\
\text { ' Ley No } 37 \text { Ley de Gestión } \\
\text { Ambiental (1999) } \\
{ }^{8} \text { Ley de Aguas Nº } 339 \text { (2004) } \\
\text { Organización territorial: Provincias }\end{array}$ & $\begin{array}{l}{ }^{8} \text { La Secretaría Nacional de Agua (SENAGUA) ejerce desde octubre del } \\
2008 \text { la rectoría nacional en la gestión y administración de los recursos } \\
\text { hídricos. } \\
\text { Entidades relacionadas con el recurso hídrico: } \\
{ }^{8} \text { Ministerio de Desarrollo Urbano y Vivienda (MIDUVI), con su } \\
\text { Subsecretaría de Agua Potable, Alcantarillado y Residuos. } \\
{ }^{8} \text { Instituto Nacional de riego y Drenaje (INAR) } \\
{ }^{8} \text { Banco del Estado (BEDE) }\end{array}$ \\
\hline Paraguay & $\begin{array}{l}\text { Legislación: } \\
\text { ' Gran dispersión de disposiciones } \\
\text { legales y serias inconsistencias. } \\
\text { Ley No 294, Evaluación de Impacto } \\
\text { Ambiental (1993) } \\
\text { Ley No } 156 \mid \text { de creación del } \\
\text { Sistema Nacional del Ambiente } \\
(2000) \\
{ }^{15} \text { Ley No 3239. Ley de los Recursos } \\
\text { Hídricos del Paraguay (2007) } \\
\text { Organización territorial: } \\
\text { Departamento }\end{array}$ & $\begin{array}{l}\text { ' La gestión de los recursos hídricos se realiza con un enfoque sectorial } \\
\text { y las instituciones del sector hídrico están fuertemente fragmentadas y } \\
\text { ninguna de ellas tiene asignado ni ejerce la responsabilidad de ente rector } \\
\text { de la política, planificación y gestión integrada de los recursos hídricos. } \\
{ }^{15} \text { La Secretaría del Ambiente (SEAM) es la autoridad encargada de la } \\
\text { aplicación de la Ley N } N^{\circ} 3239 \text {. } \\
\text { Entidades relacionadas con el recurso hídrico: } \\
\text { 'Consejo Nacional del Ambiente (CONAM) } \\
\text { Secretaría del Ambiente (SEAM) } \\
\text { Dirección General de Protección y Conservación de los Recursos } \\
\text { Hídricos, que forma parte de la SEAM. }\end{array}$ \\
\hline Perú & $\begin{array}{l}\text { Legislación: } \\
\text { I } 6 \text { Ley General de Aguas (2009) } \\
\text { Organización territorial: } \\
\text { Departamentos }\end{array}$ & $\begin{array}{l}{ }^{16} \text { Autoridad Nacional del Agua, ente rector y máxima autoridad técnico- } \\
\text { normativa del Sistema Nacional de Gestión de Recursos Hídricos, el cual } \\
\text { es parte del Sistema Nacional de Gestión Ambiental. } \\
\text { Entidades relacionadas con el recurso hídrico: } \\
{ }^{16} \text { Autoridad Nacional del Agua (ANA) } \\
\text { I Instituto Nacional de Desarrollo (INADE), dependiente del Ministerio } \\
\text { de la Presidencia. }\end{array}$ \\
\hline Uruguay & $\begin{array}{l}\text { Legislación: } \\
\text { ' Decreto } N^{\circ} 26 \text { l de creación de } \\
\text { la Comisión Técnica Asesora de la } \\
\text { Protección del Medio Ambiente } \\
\text { (COTAMA) (I993) } \\
\text { Ley N } N^{\circ} 14859 \text { Código de Aguas } \\
\text { (1978) } \\
\text { 17 Ley N } N^{\circ} 18.610 \text { Política Nacional } \\
\text { de Aguas (2009) } \\
\text { Organización territorial: } \\
\text { Departamentos }\end{array}$ & $\begin{array}{l}\text { ' No existe un organismo responsable de coordinar el ordenamiento y } \\
\text { desarrollo de los recursos hídricos y las políticas intersectoriales a nivel } \\
\text { nacional. } \\
\text { La Comisión Técnica Asesora de la Protección del Medio Ambiente } \\
\text { (COTAMA) es una instancia de consulta en el tema de medio ambiente. } \\
\text { Entidades relacionadas con el recurso hídrico: } \\
{ }^{17} \text { Dirección Nacional de Aguas y Saneamiento (DINASA) } \\
\text { ' Comisión Técnica Asesora de la Protección del Medio Ambiente } \\
\text { (COTAMA) }\end{array}$ \\
\hline
\end{tabular}




\begin{tabular}{|c|c|c|}
\hline País & $\begin{array}{l}\text { Legislación en materia del agua y } \\
\text { organización territorial }\end{array}$ & Marco Institucional y administrativo del recurso hídrico \\
\hline Venezuela & $\begin{array}{l}\text { Legislación: } \\
\text { No se registra. } \\
\text { Organización territorial: Estados }\end{array}$ & $\begin{array}{l}\text { 1,18 La gestión de los recursos hídricos se realiza de manera centraliza- } \\
\text { da por el Ministerio del Ambiente. Le corresponde como Autoridad } \\
\text { Nacional de Aguas, la planificación, administración, aprovechamiento, } \\
\text { regulación y control de los recursos hídricos, sin embargo, su capacidad } \\
\text { institucional y financiera ha sido muy limitada. } \\
\text { Entidades relacionadas con el recurso hídrico: } \\
{ }^{18} \text { Ministerio del Ambiente. } \\
\text { Consejo Nacional de Aguas. } \\
\text { Vice Ministerio del Agua. } \\
\text { Consejos de Región Hidrográfica. } \\
\text { Consejos de Cuenca. }\end{array}$ \\
\hline
\end{tabular}

Fuentes: I: Jouravlev, 200 I. 2: SSRH, 2006. 3: Van Damme, 2002. 4: Landgraf de Siqueira \& Raquel de Carvalho, 20I0. 5: Matus et al., 2004. 6: MAVDT, 20 10. 7: INRH, 20II. 8: Rojas, 20 I0a. 9: Larios, 20 I0. I0: Arriola \& Ocón, 20। I. II: Rodríguez, 20 I I. 12: CONAGUA, 2006. 13: Arriola \& Ocón, 20 I0. 14:Trejos, 20 I I. I 5: Benítez, 20 I I. I6: Rojas, 2010 b. 17: Bellagamba, 20 I I. 18: Dantant, 201 I.

\section{Bibliografía}

Aldama, A. \& Gómez, L. (1996). Capacity Building for the Mexican Water Sector Through Research, Development, Training and Education. En: Water Resources Assessment and Management Strategies in Latin America and the Caribbean, Proceedings of the WMO/IDB Conference, San José, Costa Rica.

Arriola, C. \& Ocón, L. (20I I). Recursos Hídricos - Guatemala 2011. Centro del Agua para América Latina y el Caribe. Guatemala. 70 p. Consultado: 28 jul. 2011. Obtenido desde: http://centrodelagua.org/plataforma/informes/ Guatemala20 I I Final.pdf

Arriola, C. \& Ocón, L. (20 I0). Recursos Hídricos - Nicaragua 2010. Centro del Agua para América Latina y el Caribe. Nicaragua. 47 p. Consultado: 01 ago. 201 I. Obtenido desde: http://centrodelagua.org/plataforma/informes/FichaTecnicaNicaragua. pdf

Asociación Mundial para el Agua, Centroamérica. (2006). Situación de los Recursos Hídricos en Centroamérica: Hacia una Gestión Integrada. Ed. Virginia Reyes G. 3ra. ed. San José: GWP-CA. 100 p.

Astorga, Y. (2009). Situación del recurso hídrico. Ponencia preparada para el Decimoquinto Informe Estado de la Nación. San José: Programa Estado de la Nación, 39 p.

Avila, P. (2002). Cambio Global y Recursos Hídricos en México: Hidropolítica y Conflictos Contemporáneos por el agua. Instituto Nacional de Ecología. México. 107 p. Consultado: 20 ago. 2011. Obtenido desde: http://www.ine.gob.mx/descargas/ cuencas/cambio_global_y_rec_hdricos_mex.pdf

Ballestero, M., Brown, E., Jouravlev, A., Küffner, U. \& Zegarra, E. (2005). Administración del Agua en América Latina: situación actual y perspectivas. Serie Recursos Naturales e Infraestructura, CEPAL, No 90. Santiago, 80 p.
Bellagamba, J. (201 I). Recursos Hídricos - Uruguay 201 I. Centro del Agua para América Latina y el Caribe. Uruguay. 92 p. Consultado: 03 ago. 201 I. Obtenido desde: http://centrodelagua.org/plataforma/informes/Uruguay20 I I Final.pdf

Benítez, E. (201 I). Recursos Hídricos - Paraguay 201 I. Centro del Agua para América Latina y el Caribe. Paraguay. 69 p. Consultado: 03 ago. 20 I I. Obtenido desde: http://www.centrodelagua.org/plataforma/informes/Paraguay20 I I Final.pdf

CCAD (Comisión Centroamericana de Ambiente y Desarrollo), CAC Consejo Agropecuario Centroamericano) \& SISCA (Secretaría de Integración Social Centroamericana). (2006). Estrategia Centroamericana de Gestión Integrada de Recursos Hídricos. 23 p.

CEPAL (Comisión Económica para América Latina y el Caribe). (2005). Los Recursos Hídricos y la Agricultura en el Istmo Centroamericano. México. 77 p.

CEPAL (Comisión Económica para América Latina y el Caribe). (2007). CEPAL advirtió sobre la escasez de agua en América Latina. Diario Cooperativa. Santiago, Cl. Mar. Consultado: 04 ago. 201 I. Obtenido desde: http://www.cooperativa.cl/p4_ noticias/site/artic/20070322/pags/20070322 I 25247.html

CONAGUA (Comisión Nacional del Agua). (2006). Gestión Integrada de los Recursos Hídricos en México. Veracruz. 37 p. Consultado: 01 ago. 201 I. Obtenido desde: http://portal.veracruz.gob.mx/pls/portal/docs/PAGE/GOBVERSFP/ SFPPDIFUSION/SFPOTRASPUBLICACIONES/ SFPFORODELAGUA2006/3.\%20GESTI_N\%20 INTEGRADA\%20DEL\%20AGUA\%20EN\%20M_XICO.PDF

CONAGUA (Comisión Nacional del Agua) (2010). Estadísticas del Agua en México, edición 20 10. México. 258 p. Consultado: 21 ago. 201l. Obtenido desde: http://www.conagua.gob. mx/CONAGUA07/Publicaciones/Publicaciones/EAM20 I0| 6Junio2010.pdf 
Dantant, R. (201 I). Recursos Hídricos - Venezuela 201 I. Centro del Agua para América Latina y el Caribe. Venezuela. 68 p. Consultado: 03 ago. 201 I. Obtenido desde: http://centrodelagua.org/plataforma/informes/Venezuela20 I I Final.pdf

Dourojeanni, A. (2000). Desafíos para la gestión integrada de los recursos hídricos. Policy and Institutions for Integrated Water Resources Management. International Water Resources Association (IWRA), 19 p.

Dourojeanni, A. \& Jouravlev, A. (200I). Crisis de Gobernabilidad en la Gestión del Agua. Serie Recursos Naturales e Infraestructura, CEPAL. Santiago, Chile. 83 p.

Dourojeanni, A. \& Jouravlev, A. (2002). Evolución de Políticas Hídricas en América Latina y el Caribe. Serie Recursos Naturales e Infraestructura, CEPAL. Santiago, Chile. 74 p.

ESRI (1998). Esri Profile of the Content Standard for Digital Geospatial Metadata. New York, USA. Consultado: 12 jun. 20 I I. Obtenido desde: http://www.esri.com/metadata/esriprof80.html\#Top

FAO (Organización de las Naciones para la Agricultura y la Alimentación). (2000). Perspectivas Regionales. Resumen General de América Latina y el Caribe. Sistema de información de la FAO sobre el agua y la agricultura. Aquastat. Consultado: 19 ene. 201 I. Obtenido desde: http://www.fao. org/nr/water/aquastat/regions/indexesp.stm

García, L. (1998). Manejo integrado de los recursos hídricos en América Latina y el Caribe. Washington D.C. No. ENV-23. Obtenido desde: http://www.bvsde.paho.org/bvsarg/e/fulltext/caribe/caribe.pdf

Guzmán, I. (2008). Recursos hídricos en América Latina: planificación... es la estrategia. Tecnología en Marcha, Vol. 21-I, Enero-Marzo 2008, pp. |6|-173.

INRH (Instituto Nacional de recursos Hidráulicos). (20II). Recursos Hídricos - Cuba 2011. Centro del Agua para América Latina y el Caribe. Cuba. 42 p. Consultado: 27 jul. 201 I. Obtenido desde: http://centrodelagua.org/plataforma/ informes/Cuba20 I I Final.pdf

Jouravlev, A. (200 I). Administración del agua en América Latina y el Caribe en el umbral del siglo XXI. Serie Recursos Naturales e Infraestructura, CEPAL. Santiago, Chile. 72 p.

Landgraf de Siqueira, R. \& Raquel de Carvalho, M. (2010). Gestión de Recursos Hídricos y Costeros en Brasil. Curso Regional en Manejo Integrado de Aguas y Áreas Marino Costeras: Teoría y Práctica. Ministerio de Medio Ambiente, Brasil. 39 p. Consultado: 21 jul. 201 I. Obtenido desde: http:// www.pnuma.org/agua-miaac/REGIONAL/MATERIAL\%20 ADICIONAL/PRESENTACIONES/PARTICIPANTES/ BRASIL/Recursos\%20Hidrico\%20BRASIL.pdf

Larios, S. (2010). Construyendo la Política del Recurso Hídrico en El Salvador hacia la Gobernabilidad en la Gestión del Agua. El Salvador, Ministerio de Medio Ambiente y Recursos
Naturales. 25 p. Consultado: 28 jul. 20 I I. Obtenido desde: http://www.marn.gob.sv/phocadownload/politica_gestion_ integral_recursos_hidricos_MARN.pdf

Matus, N., Fernández, B., Aedo, M.P. \& Larraín, S. (2004). Recursos Hídricos en Chile: Desafios para la Sustentabilidad. Chile. 172 p. Consultado: 27 jul. 20 I I. Obtenido desde: www.chilesustentable.net/web/wp-content/plugins/downloads-manager/ upload/RECURSOSHIDRICOS.pdf

MAVDT (Ministerio de Ambiente, Vivienda y Desarrollo Territorial). (20 I0). Política Nacional para la Gestión Integral del Recurso Hídrico. Colombia. 124 p. Consultado: 27 jul. 2011. Obtenido desde: www.humboldt.org.co/iavh/documentos/politica/politicas_ambientales/2010\%20Politica\%20 Nacional\%20Gestion\%20Recurso\%20Hidrico.pdf

Rodríguez, J. (201 I). Recursos Hídricos - Honduras 201 I. Centro del Agua para América Latina y el Caribe. Honduras. 96 p. Consultado: 28 jul. 20ll. Obtenido desde: http://centrodelagua.org/plataforma/informes/Honduras20 I I Final.pdf

Rojas, F. (2010a). Recursos Hídricos - Ecuador 2010. Centro del Agua para América Latina y el Caribe. Ecuador. 48 p. Consultado: 28 jul. 20ll. Obtenido desde: http://centrodelagua.org/plataforma/informes/FichaTecnicaEcuadorFinal. pdf

Rojas, F. (20 I Ob). Recursos Hídricos - Perú 20 I 0. Centro del Agua para América Latina y el Caribe. Perú. 40 p. Consultado: 03 ago. 20 I I. Obtenido desde: http://centrodelagua.org/plataforma/informes/FichaTecnicaPeruFinal.pdf

SSRH (Subsecretaría de Recursos Hídricos). (2006). Descripción del Proceso de Planificación. Avances Hasta la Fecha. Ministerio de Planificación Federal, Inversión Pública y Servicios de Argentina. Consultado: 21 jul. 201 I. Obtenido desde: http:// www.hidricosargentina.gov.ar/PlanBoton l.html

Trejos, N. (20I I). Recursos Hídricos - Panamá. (20l I). Centro del Agua para América Latina y el Caribe. Panamá. 63 p. Consultado: 03 ago. 201l. Obtenido desde: http://centrodelagua.org/plataforma/informes/Panama20 I I Final.pdf

Valencia, J., Díaz, J. \& Ibarrola, H. (2004). La Gestión Integrada de los Recursos Hídricos en México: nuevo paradigma en el manejo del agua. En: Cotler, H. (compilador). Manejo Integral de Cuencas en México. México, D.F.: Instituto Nacional de Ecología. pp 201-209.

Van Damme, P. (2002). Disponibilidad, Uso y Calidad de los Recursos Hídricos en Bolivia. 90 p. Consultado: 21 jul. 201 I. Obtenido desde: http://revistavirtual.redesma.org/vol I 0/pdf/ informacion/recursos_hidricos_bol.pdf

Van Hofwegen, P. \& Jaspers, F. (2000). Marco analítico para el manejo integrado de recursos hídricos. Lineamientos para la evaluación de marcos institucionales. Washington D.C. 92 p. Obtenido desde: http://cidbimena.desastres.hn/docum/Honduras MarcoAnaliticoparael ManejolntegradodelosRecursosHidricos.pdf 